\title{
Space Charge Dynamics in Silica-based Polyethylene Nanocomposites
}

\author{
Yan Wang, George Chen and Alun Vaughan \\ The Tony Davies High Voltage Laboratory, University of Southampton \\ Southampton SO17 1BJ, United Kingdom \\ *E-mail: yw14g13@ecs.soton.ac.uk
}

\begin{abstract}
Nanodielectrics have been investigated recently as they have shown some improved dielectric properties that are important for high voltage insulation applications. One of these improvements is the reduction in space charge when the nanocomposites are subjected to high dc electric fields. It has been widely reported that the inclusion of nanoparticles in polyethylene can suppress the formation of space charge in the material. However, the detailed mechanisms that are responsible for the charge suppression are not known. In the present paper, charge dynamics in nanosilica based polyethylene nanocomposites have been observed using the pulsed electroacoustic technique. A range of electric fields have been applied to the nanocomposites with different nanosilica loading ratios to investigate the impact of the applied electric field on charge suppression. In addition the effect of surface treatment of nanosilica particles on charge dynamics has also been studied by comparing treated and untreated nanocomposites with the same loading ratio of nanosilica particles. The influence of charge dynamics, especially at higher applied electric fields, on electric breakdown of these nanocomposites has been discussed.
\end{abstract}

\section{INTRODUCTION}

Space charge formation is regarded as one of most important factors which can produce significant influence on the overall dielectric properties of polymeric insulation system [1]. To be more specific, space charge can modify the electric field in the dielectric materials as a function of time. This may lead to material degradation and premature failure. Generally speaking, the formation of space charge at higher electric fields can be attributed to the difference of injection rate and removal rate in the material. Charge injection and trapping are strongly related to the nature of the material. It has been reported that nanodielectrics can suppress space charge injection, therefore, reduction in charge accumulation [2]. Furthermore, for nanocomposites, the amount of internal charge is not only less but the rate of charge decay is also much faster comparing with unfilled polymer [3]. In addition, the space charge behavior of polymer filled with surface treated nanoparticles which is used for a better dispersion has been investigated [4]. Space charge dynamics in nanocomposites is related to the other important dielectric properties such as DC breakdown [5].

In this paper, a blend polyethylene which includes $80 \%$ LDPE and 20\% HDPE is used as the polymer matrix filled with nanosilica with both untreated surface and trimethoxy(propyl)silane treated. Samples with three loading ratios of nanosilica, i.e. $2 \mathrm{wt} \%, 5 \mathrm{wt} \%$ and $10 \mathrm{wt} \%$ were prepared and space charge dynamics in three loading ratios of nanocomposites samples have been measured under the three applied electric fields, i.e. $30 \mathrm{kV} / \mathrm{mm}, 50 \mathrm{kV} / \mathrm{mm}$ and $70 \mathrm{kV} / \mathrm{mm}$.

\section{EXPERIMENT DETAILS}

LDPE used in this research is grade LD100BW which was obtained from the ExxonMobil Chemicals, and HDPE grade Rigidex HD5813A was obtained from the BP Chemicals. The nanosilica selected from Sigma-Aldrich, and the size of this nanopowder ranges from $10 \mathrm{~nm}$ to $20 \mathrm{~nm}$. For these nanosilica which is not accept treated by using trimethoxy(propyl)silane, is named untreated nanosilica in this research. Meanwhile for the nanosilica which is functionalized by utilizing trimethoxy(propyl)silane, is named C3 treated nanosilica [6]. Similar sample preparation procedure in [6] has been adopted. The samples with two different thicknesses of $\sim 85 \mu \mathrm{m}$ and $\sim 120 \mu \mathrm{m}$ were pressed at $150^{\circ} \mathrm{C}$ and isothermal crystallized at $115^{\circ} \mathrm{C}$ in oil bath. Thinner samples were used for DC breakdown test due to the limitation of HVDC supply and thicker samples were for space charge measurements.

The pulsed electro-acoustic (PEA) technique was applied for measuring space charge behaviors [7]. The pulse generator has an amplitude of $600 \mathrm{~V}$ and a pulse width of $5 \mathrm{~ns}$. A very thin layer of silicone oil was placed on both sides of sample to achieve a better acoustic transmission. Semiconducting polymer was used as top electrode for better acoustic impedance match and aluminum as bottom electrode in the PEA system. For all the charge measurements, the calibration was conducted at $10 \mathrm{kV} / \mathrm{mm}$. For each sample charge dynamics were monitored over a period of 2 hours upon the voltage application at room temperature. For DC electrical breakdown test was placed between two ball bearing electrodes immersed in silicone oil. The voltage ramping rate of DC breakdown test was $100 \mathrm{~V} / \mathrm{s}$. 20 breakdown data were typically obtained and they were analyzed using the Weibull distribution [8].

\section{A. Space charge dynamics}

\section{RESULTS}

It is worth to point out that breakdown occurred for $10 \mathrm{wt} \%$ untreated and 10wt\% C3 treated samples when measuring charge accumulation at $50 \mathrm{kV} / \mathrm{mm}$. It has been repeated several 
times for avoiding randomness and faulty samples. The time to failure for $10 \mathrm{wt} \%$ untreated samples was about 10 minutes and 50 minutes for $10 \mathrm{wt} \% \mathrm{C} 3$ treated samples. Therefore, space charge data is not completed for both $10 \mathrm{wt} \%$ untreated and $10 \mathrm{wt} \% \mathrm{C} 3$ treated samples at $50 \mathrm{kV} / \mathrm{mm}$ or above. Figure 1 shows charge dynamics and their decay in unfilled samples at $30 \mathrm{kV} / \mathrm{mm}, 50 \mathrm{kV} / \mathrm{mm}$ and $70 \mathrm{kV} / \mathrm{mm}$. At $30 \mathrm{kV} / \mathrm{mm}$, charge injection of both polarities can clearly be seen in Figure 1 (a1). This is further confirmed by the removing capacitive charge of the applied electric field as shown in Figure 1 (a2). When the applied electric field increases to $50 \mathrm{kV} / \mathrm{mm}$, it seems that the heterocharge develops near to the cathode as shown in Figure 1 (b1). The origin of heterocharge formation is often considered from the ionization of additives or impurities. In the present case, considering the results at $30 \mathrm{kV} / \mathrm{mm}$, it is believed that the heterocharges near the cathode come from injected charges from the anode. The charges move very fast and reach to the cathode in a very short period of time. Negative charge injection still takes place at the cathode and the amount of injection may not be as strong as that positive charge. The above argument is supported by injected charge distribution after the removal of capacitive charge caused by the applied electric field as shown in Figure 1 (b2) where the injected negative charge is readily observable near to the cathode. The situation at $70 \mathrm{kV} / \mathrm{mm}$ is very similar to that at $50 \mathrm{kV} / \mathrm{mm}$ but more complex in the middle of the sample.

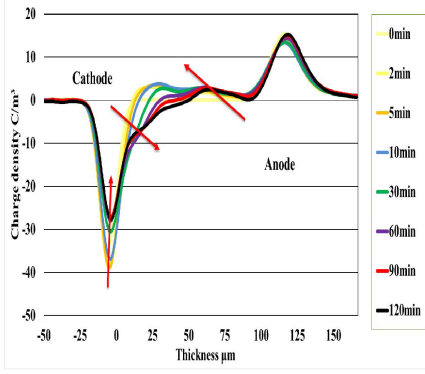

(a1)

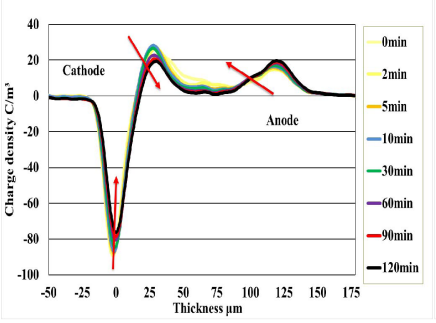

(b1)

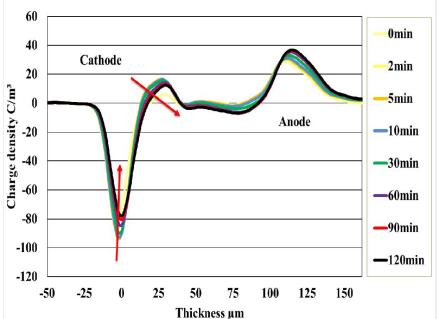

(c1)

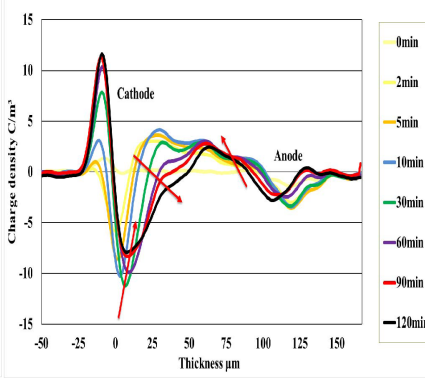

(a2)

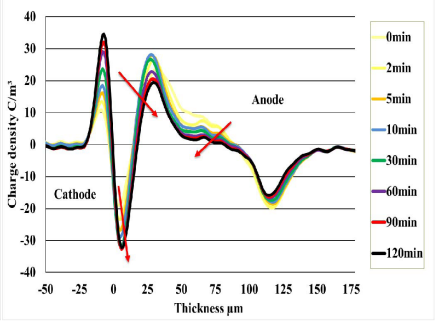

(b2)

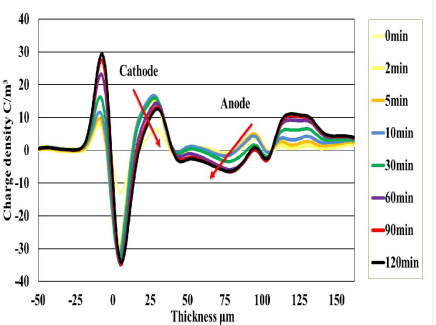

(c2)
Figure 1: Space charge dynamics in unfilled samples (a1) charge distribution at $30 \mathrm{kV} / \mathrm{mm}$, (a2) injected charge, (b1) charge distribution at $50 \mathrm{kV} / \mathrm{mm}$, (b2) injected charge, $(\mathrm{c} 1)$ charge distribution at $70 \mathrm{kV} / \mathrm{mm},(\mathrm{c} 2)$ injected charge.

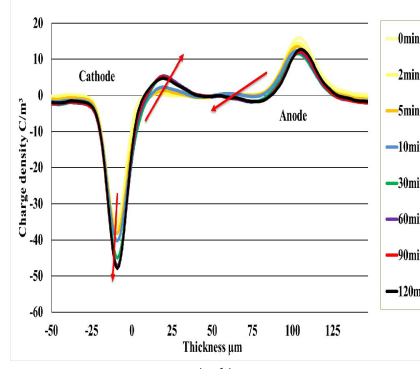

(a1)

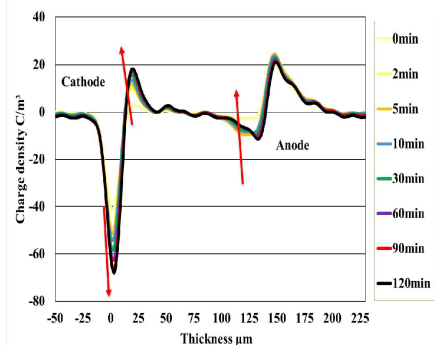

(b1)

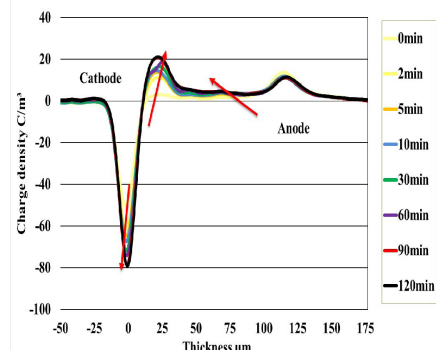

(c1)

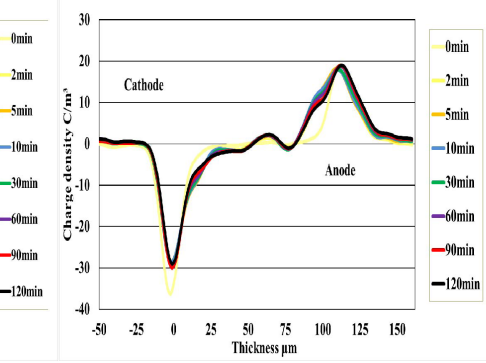

(a2)

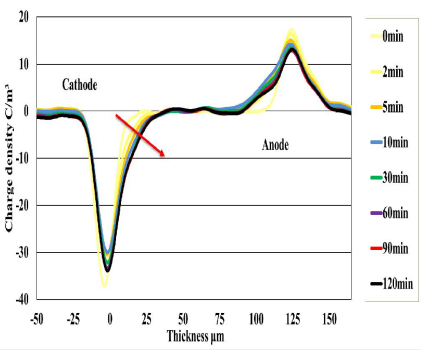

(b2)

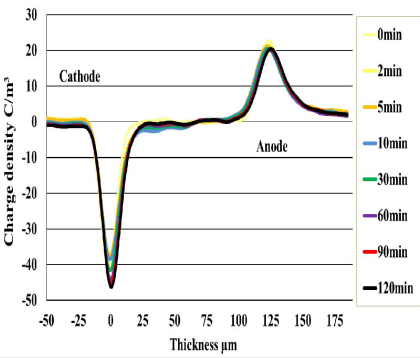

(c2)
Figure 2: Space charge behaviors at $30 \mathrm{kV} / \mathrm{mm}$ (a1) $2 \mathrm{wt} \%$ untreated (a2) $2 \mathrm{wt} \%$ $\mathrm{C} 3$ treated (b1) $5 \mathrm{wt} \%$ untreated (b2) $5 \mathrm{wt} \% \mathrm{C} 3$ treated (c1) $10 \mathrm{wt} \%$ untreated (c2) $10 \mathrm{wt} \% \mathrm{C} 3$ treated.

Figure 2 shows the space charge behavior of both $2 \mathrm{wt} \%$ untreated and C3 treated samples. Comparing with Figure 1 (a1), it seems that untreated nanocomposites intend to strengthen positive charge injection, resulting in positive charge peak near to the cathode due to high mobility. The amount of positive charge increases with the weight percentage of untreated nanosilica in the sample. On the other hand, C3 treated nanocomposites can effectively suppress charge injection, especially for positive charge. Charge distributions in nanocomposites at $50 \mathrm{kV} / \mathrm{mm}$ are shown in Figure 3. Overall, they show same trend as that at $30 \mathrm{kV} / \mathrm{mm}$ except the effect being amplified. Charge injection intends to increases non-linearly with the electric field. As a result, the breakdown occurs for both $10 \mathrm{wt} \%$ nanocomposite samples. Because of strong positive charge injection, untreated $10 \mathrm{wt} \%$ sample fails at much short time. Figure 4 shows the injected space charge behavior of $10 \mathrm{wt} \%$ untreated and $10 \mathrm{wt} \% \mathrm{C} 3$ treated. Larger positive charge peak close to the cathode is believed to be the cause of an easy breakdown for $10 \mathrm{wt} \%$ untreated samples because the electric field enhancement at the cathode will lead to more negative charge injection, resulting in a large current. The negative charge injection is evident from the negative peak in the middle of the sample 
adjacent to the positive peak. On the other hand, the formation of homocharge occurs in $10 \mathrm{wt} \% \mathrm{C} 3$ treated samples due to the higher applied electric field.

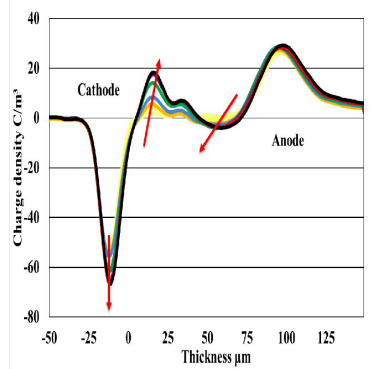

(a1)

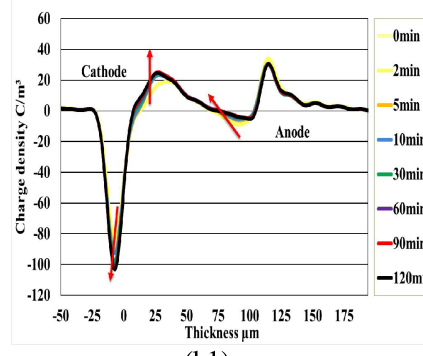

(b1)

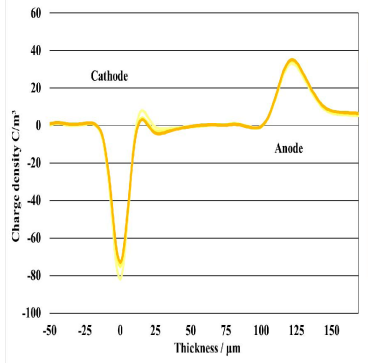

(c1)

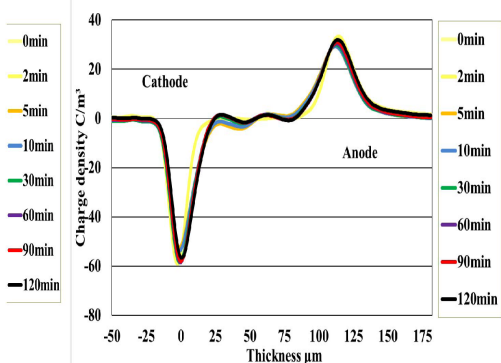

(a2)

(b2)

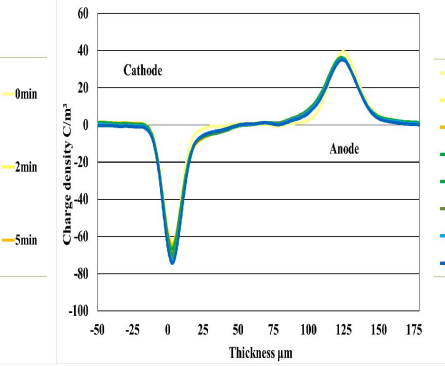

(c2)

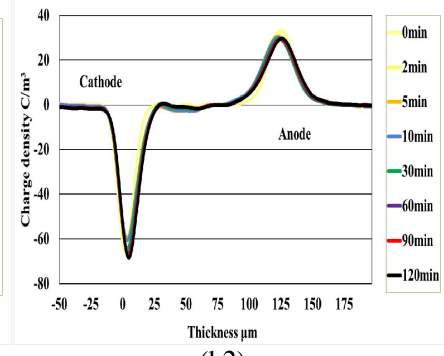

Figure 3: Space charge behaviors at $50 \mathrm{kV} / \mathrm{mm}$ (a1) $2 \mathrm{wt} \%$ untreated (a2) $2 \mathrm{wt} \%$ $\mathrm{C} 3$ treated (b1) $5 \mathrm{wt} \%$ untreated (b2) $5 \mathrm{wt} \% \mathrm{C} 3$ treated (c1) $10 \mathrm{wt} \%$ untreated (c2) $10 \mathrm{wt} \% \mathrm{C} 3$ treated.

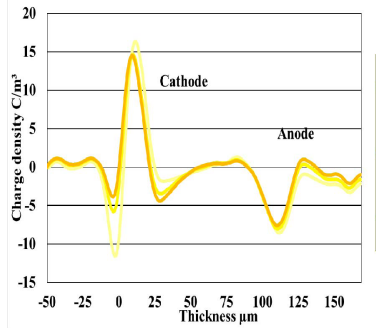

(a)

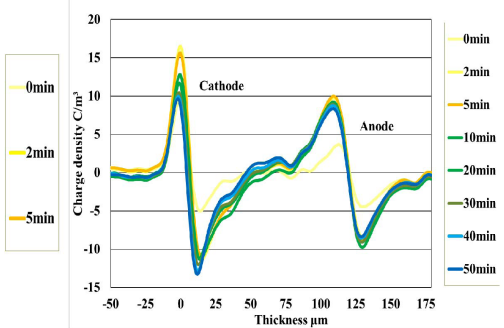

(b)
Figure 4: Injected space charge behavior stressed at $50 \mathrm{kV} / \mathrm{mm}$ (a) $10 \mathrm{wt} \%$ untreated (b) $10 \mathrm{wt} \% \mathrm{C} 3$ treated

When the applied electric field increases to $70 \mathrm{kV} / \mathrm{mm}$ charge the injection becomes so strong and two different polarity of charge interact with each other such as recombination etc. This is especially true for untreated samples. Negative charge peak occurs in the middle part of the samples. Charge injection for C3 treated samples is also obvious.

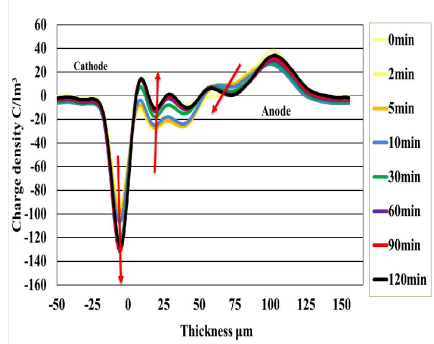

(a1)

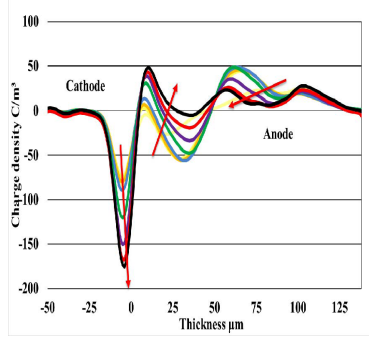

(b1)

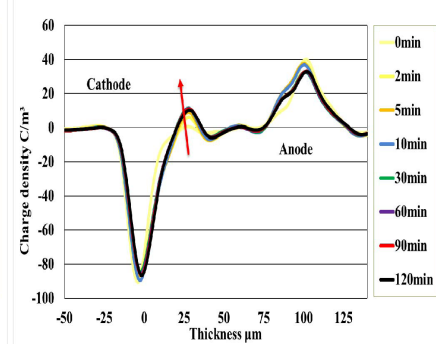

(a2)

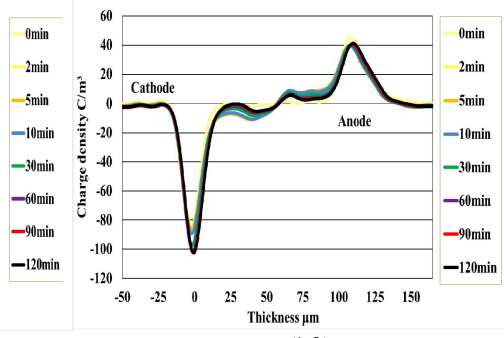

(b2)
Figure 5: Space charge behavior stressed at $70 \mathrm{kV} / \mathrm{mm}$ (a1) $2 \mathrm{wt} \%$ untreated (a2) $2 \mathrm{wt} \% \mathrm{C} 3$ treated (b1) $5 \mathrm{wt} \%$ untreated (b2) $5 \mathrm{wt} \% \mathrm{C} 3$ treated.

\section{B. DC breakdown}

A typical Weibull breakdown plot is shown in Figure 6 from which both characteristic breakdown strength and slope can be obtained.

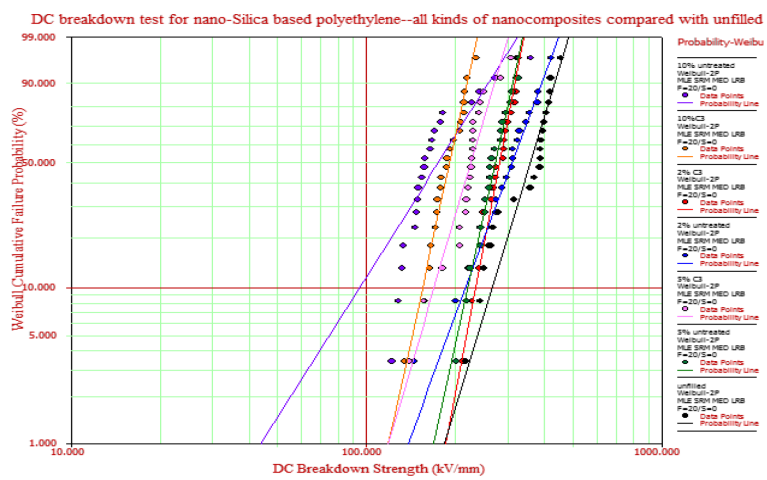

Figure 6: DC breakdown test for all nanocomposites compared with unfilled polyethylene

Significant differences in breakdown strength due to inclusion of nanosilica can be seen from Figure 6. The impact of surface treatment on DC breakdown strength can also be seen and the details are summarized in Table 1.

\begin{tabular}{|c|c|c|c|c|}
\hline Sample & $\beta$ & a lower bound & $\eta \mathrm{kV} / \mathrm{mm}$ & b upper bound \\
\hline Unfilled & 6.38 & 355.02 & 378.83 & 402.83 \\
\hline $2 w t \%$ untreated & 5.23 & 307.42 & 332.58 & 358.44 \\
\hline $2 \mathrm{wt} \% \mathrm{C} 3$ treated & 10.17 & 280.38 & 291.98 & 303.45 \\
\hline $5 w t \%$ untreated & 8.89 & 270.94 & 283.83 & 296.62 \\
\hline 5wt $\%$ C3 treated & 6.56 & 224.11 & 238.55 & 253.27 \\
\hline $10 \mathrm{wt} \%$ untreated & 3.07 & 172.41 & 197.05 & 223.99 \\
\hline $10 \mathrm{wt} \% \mathrm{C} 3$ treated & 8.82 & 190.21 & 199.34 & 208.4 \\
\hline
\end{tabular}


DC breakdown strength of nanocomposites is lower than the unfilled polyethylene and functionalization of C3 does not have the positive effect on the DC breakdown performance. The performance of $2 \mathrm{wt} \%$ and $5 \mathrm{wt} \%$ C3 treated nanocomposites is inferior comparing with untreated nanocomposites. However, with $10 \mathrm{wt} \%$ loading ratio, the performance of $\mathrm{C} 3$ treated approaches the untreated.

\section{Discussion}

Space charge dynamics of both unfilled polyethylene and nanocomposites are complicated especially under higher applied electric fields. Usually, homocharge is expected to be observed near to the electrodes. However, as the mobility of both positive and negative charges is a function of the electric field, the actual charge distribution is difficult to predict. Additionally, the movement of charge is very sensitive to the treatment of the nanosilica in nanocomposites. It seems that the positive charge injected from the anode tends to move to the cathode quickly to form heterocharges in unfilled samples as shown Figure 1 and untreated samples as shown in Figures 2 - 5. However, this does not happen in C3 treated samples, possibly due to two reasons: (i) more effective suppression of charge injection after $\mathrm{C} 3$ surface treatment and (ii) the charge mobility has been hindered by the C3 surface treatment on nanosilica. Typically, higher loading ratio results in more heavily aggregation. The $\mathrm{C} 3$ treatment is used to obtain better dispersion of nanoparticle into the nanocomposites and it is able to change the polarity of nanoparticles because the nanosilica will absorb water. According to Figure 7 depicted, the hydroxyls are attached to the surface of untreated nanosilica whereas the treated nanosilica have more methyl. Moreover, it has been reported that the shape of nanoparticle can be modified by the saline chain [9]. Space charge dynamics are sensitive to the inclusion of nanoparticles as well as its surface treatment. Higher electric field leads to more charge injection and results in complicated charge dynamics as shown in the results obtained at $70 \mathrm{kV} / \mathrm{mm}$. Positive charges and negative charges in the samples can coexist or are more convenient to recombine. Since the PEA only can measure the net charge in a material, therefore, it is difficult to analyze the detailed mechanisms of transport, trapping/detrapping and recombination processes in nanocomposites.

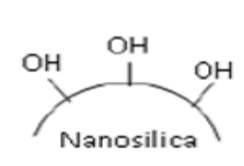

(a) untreated nanosilica

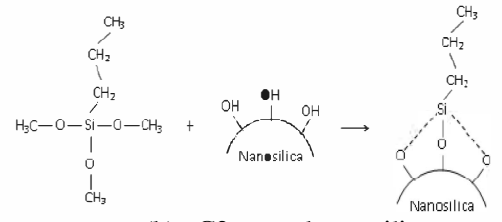

(b) $\mathrm{C} 3$ treated nanosilica
Figure 7: Reaction of the untreated nanosilica and C3 treated nanosilica [4]

From DC breakdown strength point of view, it seems that its relationship with space charge is more complex. Generally, DC breakdown strength does not depend on the net amount of space charge directly. The conduction current may be significant in the case of samples with a high loading of nanosilica particles. This will be verified in the next stage of the work. It seems that the loading ratio of nanoparticles has shown some impact on DC breakdown strength. Although all of nanocomposites possess lower DC breakdown strength compared with unfilled blend polyethylene, the breakdown strength decreases with the increase in loading ratio of nanoparticles. This is also suitable for analysis the space charge dynamic. $2 \mathrm{wt} \%$ and $5 \mathrm{wt} \%$ nanoparticle have less space charge comparing with the $10 \mathrm{wt} \%$. The purpose of functionalization of nanoparticle is to obtain better dispersion of nanoparticles. However, the DC breakdown results do not support that better dispersion leads to higher DC breakdown strength. It has been widely reported that moisture plays an important role in determining electrical performance of nanocomposites, further research is required to understand the role of moisture in nanocomposites studied in this work.

\section{CONClusion}

Generally, the lower loading ratio of nanoparticle in nanocomposites can achieve better performance for both space charge dynamic and DC electric breakdown strength. The Functionalization of nanoparticles may change the shape and behaviors of original nanoparticles which will show impact on space charge dynamics and the performance of DC breakdown strength. The surface treated nanocomposites have better dispersion and can suppress space charge especially the formation of heterocharge. At higher electric fields, space charge dynamics are more complicated. DC breakdown strength may be more related to dynamics of charge and net conduction current rather than the net amount of space charge measured by the PEA. Further work has been planned to measure conduction current as well as examine the influence of moisture.

\section{REFERENCES}

[1] T. Mizutani, "Space charge measurement techniques and space charge in polyethylene," IEEE Trans. Dielectr. Electr. Insul., vol. 1, pp. 923-933, 1994.

[2] J. K. Nelson, J. C. Fothergill, L. A. Dissado, and W. Peasgood "Towards an understanding of nanometric dielectrics," IEEE Conf. Electr. Insul. Dielectr. Phenomena, pp. 295-298, 2002.

[3] T. Tanaka, "Dielectric nanocomposites with insulating properties," IEEE Trans. Dielectr. Electr. Insul., vol. 12, pp. 914-928, 2005.

[4] K. Y. Lau, A. S. Vaughan, G. Chen, I. L. Hosier and A. F. Holt, "On nanosilica surface functionalization using different aliphatic chain length silane coupling agents" Proceeings of the international conference on solid electrics, IEEE, 896-899, 2013.

[5] G. Chen, J.W. Zhao, "Space charge and thickness dependent dc electrical" 2012 International Conference on High Voltage Engineering and Application, Shanghai, China, September 17-20, 2012 breakdown of solid dielectrics, pp. 12-15, 2012

[6] K. Y. Lau, A. S. Vaughan, G. Chen, I. L. Hosier, and A. F. Holt, "On the dielectric response of silica-based polyethylene nanocomposites," Journal. Phys. D: Appl. Phys., vol. 46, 095303, 2013.

[7] T. Takada and T. Sakai, "Measurement of electric fields at a dielectric/electrode interface using an acoustic transducer technique," IEEE Trans. Electr. Insul., vol. 18, pp. 619-628, 1983.

[8] W. Weibull, "A statistical distribution function of wide applicability," J. Appl. Mech., vol. 18, pp. 293-297, 1951

[9] Kim, K., and White, J. L. (2002). Silica surface modification using different aliphatic chain length coupling agents and their effects on silica agglomerate size and processability. Composite Interfaces, 9(6), 541556. 